\title{
Auditory event perception: The source-perception loop for posture in human gait
}

\author{
Richard E. Pastore, Jesse D. Flint, Jeremy R. Gaston, and Matthew J. Solomon \\ Binghamton University, Binghamton, New York
}

\begin{abstract}
There is a small but growing literature on the perception of natural acoustic events, but few attempts have been made to investigate complex sounds not systematically controlled within a laboratory setting. The present study investigates listeners' ability to make judgments about the posture (upright-stooped) of the walker who generated acoustic stimuli contrasted on each trial. We use a comprehensive three-stage approach to event perception, in which we develop a solid understanding of the source event and its sound properties, as well as the relationships between these two event stages. Developing this understanding helps both to identify the limitations of common statistical procedures and to develop effective new procedures for investigating not only the two information stages above, but also the decision strategies employed by listeners in making source judgments from sound. The result is a comprehensive, ultimately logical, but not necessarily expected picture of both the source-sound-perception loop and the utility of alternative research tools.
\end{abstract}

Humans exhibit extensive abilities to use sounds to identify and monitor events in their environment; these abilities are often unappreciated and, with the exception of speech, largely unexplored (see, e.g., Handel, 1989, 1995; McAdams, 1984, 1993). As examples of these abilities, auto mechanics, physicians, and even plumbers use sound to detect possible abnormalities and make preliminary diagnoses of probable cause (e.g., Jenkins, 1985). Similar diagnostic listening strategies for human gait can be effective in podiatric medicine (J. Wernick, personal communication, May 2004). Thus, although a major long-term goal of hearing research is to understand typical auditory perception, the majority of research has investigated only the perception of simple, easily specified, laboratory-created stimuli. The resulting body of fundamental research has provided important, detailed specifications of the basic functioning of the human auditory system (e.g., Hartmann, 1998; Moore, 2003; Yost, 1994), but this scientific knowledge often seems to have limited direct relevance for understanding the ability to recognize the nature of complex natural acoustic source events. Although this situation could arise because the existing principles represent too low a conceptual level (or level of organization) to be relevant, it is at least equally likely that we lack sufficient understanding of natural source-event perception to see the relevance. The present study is designed from this perspective.

Speech is the one example of a class of complex natural source events that has been studied extensively in terms of the relationship between the properties of production (the source event) and the sounds produced, as well as in terms of the links between possible invariant acoustic cues and perceptual categories. As a source event, speech is com- plex and highly variable, yet it is characterized by relatively simple, discrete feature categories. If simple source features mapped in a relatively straightforward manner to sound, the basis for listener perception of the speech features would have been easy to identify. One hallmark of more than half a century of speech research, however, has been the failure to identify invariant acoustic cues for features of production (see, e.g., Raphael, 2005). Rejecting the possibility of a complex mapping between source and sound, speech perception is often assumed to involve some form of a highly specialized, closed module (e.g., Liberman \& Mattingly, 1985), possibly one initially shaped by early experience (e.g., Werker \& Tees, 1999). If valid, this supposition of a unique speech perception mechanism would mean that the body of research on speech would not be relevant to understanding the perception of other types of natural acoustic events.

One alternative perspective, based upon Gibson's ecological conceptualization (Gibson, 1966, 1979), is that the dynamic structure not only of speech, but of all natural acoustic events, determines the structure of the sounds produced, which in turn allows direct perception of the source event (see, e.g., Fowler, 1991; Fowler \& Rosenblum, 1991a, 1991b; Gaver, 1993; Vanderveer, 1980). With the assumption of a relatively straightforward mapping from dynamic aspects of source structure to dynamic sound structure, an easy recovery of the source event should then be possible. From such a supposition, typical studies in ecological acoustics seek to demonstrate that listeners can accurately identify properties of a source event (e.g., McAdams, Chaigne, \& Roussarie, 2004), yet these studies often provide only limited specification of 
the properties and structure of the acoustic signal (e.g., Kunkler-Peck \& Turvey, 2000). An alternative to the strict ecological perspective is that listeners may learn about basic relationships between the (sometimes complex) attributes of sounds and the source-event properties that allow either identification of or inferences about aspects of the source event (see, e.g., Mirman, Holt, \& McClelland, 2004; Wildes \& Richards, 1988). Whatever one's theoretical beliefs, a clear, and probably reasonable, assumption remains that properties of the source event are reflected in the sounds produced, and that this acoustic information must be crucial to the ability to perceive or identify the original source event. However, a real possibility also exists that source-sound relationships can be complex and variable, rather than simple and invariant.

In recent years, a small but growing number of important studies have begun to investigate specific aspects of the perception of complex auditory events. These studies have varied significantly in goals, and thus in both the research techniques employed and the answers found. Some studies have investigated the broad classification or grouping of large sets of diverse natural and artificial sounds (e.g., Ballas, 1993; Ballas \& Mullins, 1991; Gygi, 2001; Howard \& Ballas, 1980a, 1980b, 1981). More narrowly focused studies have investigated the identification of a specific source attribute for sounds produced in the laboratory under controlled conditions (e.g., Giordano \& McAdams, 2006). Lakatos, McAdams, and Caussé (1997), for example, found that listeners could judge the geometric shape of metal and wood bars struck by a mallet, but not the cross-sectional area of metal bars (see also Lakatos, Gibson, \& Cannon, 1997). Recently, Grassi (2005) found that listeners could judge the size of wood balls dropped on a ceramic plate from a fixed distance. These studies focused on well-defined questions, but the acoustic stimuli, although complex, were produced under highly controlled laboratory conditions, and thus varied almost exclusively as a function of the source property being manipulated. As a result, the sounds should have been more source diagnostic than would be typical for natural events, and listener decisions should also have been more limited, easier, and more accurate.

A few studies have investigated specific contrasts for natural acoustic events in which the source event and the acoustic stimuli exhibited variability more typically encountered in the listening environment. Examples of acoustic source contrasts investigated in these studies have included material hardness (Freed, 1990), gender identification of humans either hand-clapping (Repp, 1987) or walking (Li, Logan, \& Pastore, 1991), and the bouncing versus breaking of objects (Warren \& Verbrugge, 1984).

Each of the many types of studies we have summarized has contributed in different ways to our understanding of natural listening. However, investigations of natural listening conditions, best approximated in the last few of the studies above, pose significant, yet still poorly understood, difficulties for research design and data analysis. The present auditory event perception research goes well beyond all of the prior studies in terms of scope, source-event and sound complexity, depth of analysis, and conceptual integration.
Our study has two primary goals: (1) the development of an effective, comprehensive framework for research investigating the perception of auditory source events, and (2) the use of that framework to begin to understand the perception of a specific source-event contrast, that of humans walking in an upright versus stooped posture.

For a number of reasons, the source event selected for investigation in the present study is human gait. In a previous study ( $\mathrm{Li}$ et al., 1991), we selected this event class because the recorded stimuli are easily recognized as being generated by humans walking (e.g., few sound effects are generated by the depicted source event). This prior research taught us a great deal (in positive and negative senses) about studying this source event. Furthermore, humans have considerable knowledge about at least some aspects of the biomechanics of human gait. For instance, both anecdotal and empirical forms of evidence (see, e.g., Farrington, 1998) have shown that listeners often accurately identify the approach of familiar individuals from the sound of their footsteps. In addition, knowledge of such biomechanics is implied by both the extensive literature on gait that has used point-light walkers (e.g., Cutting, 1978; Cutting \& Kozlowski, 1977; Cutting, Proffitt, \& Kozlowski, 1978; Johansson, 1973, 1986; Proffitt \& Kaiser, 1995; Runeson \& Frykholm, 1983) and the psychological literature on motor control and programming (Jacobs \& Shiffrar, 2005). As well, extensive medical (e.g., Braune \& Fischer, 1899/1987; Cappozzo, 1991; Weber \& Weber, 1836/1992; Winter, 1987, 1989; Yamaguchi, Pandy, \& Zajac, 1991; Zatsiorsky, 1998) and podiatric (e.g., Wernick, 1993) literatures on the biomechanics of human gait have helped guide our specification of the source event.

\section{Information Triangle and the Source-Perception Loop}

In contrast to research with simple laboratory stimuli, investigations of natural events are complex and need to consider several data sets. Auditory event perception involves three different, but clearly interrelated and measurable, stages of information: the source, the sound, and perception. These three types of information can be conceptualized as the three corners of an information triangle. The sound that is produced reflects both static and dynamic attributes of the source event, and the statistical relationships between the source and sound stages of information define what the ideal listener would use in distinguishing among source-event attributes. Thus, for a good understanding of event perception, one needs to investigate the attributes of the source, sound, and listener perception stages (or corners) of the information triangle, along with the actual and potential relationships among these stages that, together, define a source-soundperception loop for research.

The importance of this three-stage approach is illustrated by the contrast between the simple assumptions about variability that are reasonable in typical laboratory studies and the complicated nature of variability that must be considered in any study of more natural source events. When one is investigating limited laboratory stimuli, it is reasonable to use traditional perceptual analyses that were 
designed to identify major effects characterized by systematic variation, in a context in which variability is random and independent of the major effect. A different situation pertains in the present study, in which stimuli were generated by a number of normal, healthy humans walking in either their normal upright posture or a stooped posture. In their normal upright posture, these walkers differed in a number of uncontrolled ways, in both their static attributes (e.g., height, weight, gender, or shoe size) and their dynamic biomechanics. In a stooped posture, they differed not only in the degree of posture change, but in both the direct consequences of that change (e.g., the magnitude of change in center of gravity) and the changes in gait biomechanics used to compensate for the change in center of gravity. Because across-walker variability exists both within and across postures, there may well not be systematic main effects of posture and random variation for all other factors. Thus, the analytic tools that are effective for more controlled research investigations might do very poorly in identifying acoustic properties, and even sourceevent attributes, that reliably reflect the posture contrast between such stimuli. These conditions are probably typical of many natural source events. Because research on the perception of complex natural events has not yet effectively addressed the problems of nonrandom variability, the identification of research strategies and tools that either are or are not effective, as well as a determination of why they vary in effectiveness, is at least as important as gaining a better understanding of the source-event perception that is being investigated.

The source variability and source-sound mapping considerations also have implications for investigating both the abilities of listeners in making source judgments and the cues they use in making the judgments. In a psychophysical study with stimuli that vary solely along a single acoustic dimension, one can assume that listeners do not differ substantially in their use of the limited stimulus information that is available. Furthermore, evidence has indicated that, when making judgments about complex stimuli or events, individual listeners not only are less-than-optimal decision-makers, who are vulnerable to internal noise and do not use all of the relevant information available, but also differ, sometimes widely, in the information they do use (see, e.g., Lutfi \& Oh, 1997; Samoylenko, McAdams, \& Nosulenko, 1996). Thus, for the perception of complex natural events, the performance of an average listener may not be an accurate indicator of either the abilities or the perceptual decision strategies of actual listeners. Thus, study into the perception of natural auditory source events differs from simple laboratory investigations not only in the nature and magnitude of variability in both the source event and the sounds produced, but also in the use the listeners make of the sound properties.

Obviously, all aspects of the present study would be simpler if we selected stimuli with clear differences in acoustic properties that listeners would believe reflected the posture contrast, since this would allow the use of traditional analyses. However, such an approach would not only bias our results, it would make the study much less important. Hence, other than eliminating walkers with abnormal gait (e.g., an initial toe impact followed by the heel impact) and acoustic stimuli with either extraneous or clearly abnormal sounds, we made no attempt to systematically select stimuli, which are thus natural and complex.

\section{RECORDING AND ANALYSIS OF SOURCE EVENTS}

The first, and often neglected, step in generating a relatively complete picture of the perception of natural auditory source events is the evaluation from a theoretical perspective of the acoustically relevant properties of the source events, with this evaluation guiding measurement of the source and then of the sound properties. In the present study, our source-event manipulation was a change in walker posture, which should cause alterations in the biomechanics of gait that should be reflected in the sounds produced. The consequences of the posture change can best be understood by examining first the biomechanics of normal upright human gait, then the alterations in biomechanics needed to adapt to the posture change. An extensive research literature has focused on normal upright gait as well as on gait pathologies (Braune \& Fischer, 1899/1987; Broer, 1973; Cappozzo, 1991; Weber \& Weber, 1836/1992; Wernick, 1993; Winter, 1987, 1989; Yamaguchi et al., 1991; Zatsiorsky, 1998). Although stooped posture may be an aspect of many pathologies, pathological gaits reflect more than just the change in posture, and because the present study only examined posture change in normal, healthy walkers, this literature was of limited relevance.

The goal in human gait is to efficiently move the body forward while maintaining support, stability, and balance. In an upright gait, the back is relatively straight and the center of gravity (or point of equilibrium) is typically maintained above the hips (see Broer, 1973, for an excellent detailed discussion of normal gait mechanics). In simple terms, the impetus for forward motion is provided by a push-off from the walking surface with the rear leg, with support of the upper body then transferred to the front (planted) leg. In order to provide a new forward support base, the swing leg can only move forward by pivoting about the hip, knee, and ankle joints, all of which add forces that tend to reduce balance and stability. In the upright posture, there are a series of well-practiced, coordinated compensatory movements that result in stability, balance, and efficiency in forward movement. However, the fact that an upright gait is relatively automatic for an individual walker does not mean that the nature of the biomechanical movements will be identical across individual walkers.

The primary walking sounds in human gait are the result of shoe-floor contact as the swing foot is planted. The initial shoe-floor impact begins with this leg's partial acceptance of weight as the angled heel of the shoe makes contact with the walking surface, followed by a fuller transfer of weight associated with the impact of the shoe sole, typically with foot flexion significantly dampening the force of this impact. The remainder of the shoe-floor contact, which occurs as the formerly planted leg becomes the swing leg, consists of lifting of the heel, then the sole, and finally the toe of the foot. The typical result across 
the individual's full shoe-floor contact is a saddle-shaped temporal pattern of pressure on the walking surface that differs little across shoe types or sizes, but does differ with the weight of the walker (Galbraith \& Barton, 1970). However, the impact sounds reflect the many dynamic forces involved, coupled with the efficiency of coordinated movements that balance those forces.

The primary change from a normal upright to a stooped posture is a bending of the torso at the hips that places the center of gravity forward. As a result, the gait in the stooped posture requires that biomechanical alterations be made to compensate for the shifted center of gravity. Stability, balance, and efficiency of gait in the stooped posture depend on the nature and coordination of the biomechanical adjustments adopted; healthy walkers may adopt a number of different biomechanical strategies. One strategy that has been observed is the bending of the knees; a lowered center of gravity is probably easier to support, but the change also alters the mechanics of foot placement. There also may be a shift from a relatively automatic to a purposeful coordination of movements. One manifestation of the shift to purposeful coordination is a neglect of arm swing, which in the upright posture is an automatic compensation for hip rotation (Broer, 1973), and this tendency was observed in our walkers. Because arm swing normally compensates for hip rotation, its absence is also probably indicative of greater instability.

Several types of acoustic consequences are expected to result from the change from upright to stooped posture. There are at least two reasons that walking pace should be more rapid: The forward position of body mass increases the forward momentum created by the rear leg pushing off the floor (Broer, 1973), and to compensate for added instability, the forward moving swing leg is likely to be planted more quickly. In addition, the angle of foot placement and the force (or dampening) of foot impact are altered both by a greater bending of the knees and by the speed of placement. These and other biomechanical changes caused by the shift to a stooped posture alter the dynamic forces that produce the foot impacts, which in turn should be reflected as changes in the temporal, spectral, and amplitude properties of the sounds of these impacts. After describing the methods of producing and recording the source event (audio and visual), we will focus on (1) validation of the posture contrast manipulation and (2) evaluation of the expected biomechanical differences due to posture contrast.

\section{Method}

Participants. Initially, 22 participants ${ }^{1}$ from Binghamton University were recorded for the study: 8 of them (4 males and 4 females) from our research staff, and 14 undergraduates (6 males and 8 females) from the Binghamton University human subjects pool. For reasons we describe below, recordings from only 14 of the participants (arbitrarily labeled 1-14) were used for subsequent evaluation and analyses.

Stimuli and recording. Recording took place in a large room $(22 \times 22$ feet $)$ with a thinly carpeted concrete floor, gypsum board walls, a drop acoustic ceiling, and low background noise [typically less than $50 \mathrm{~dB}(\mathrm{C})]$. A slightly elevated $(0.5$-in.) hardwood floor ( 2.5 feet $\times 21$ feet $\times 1.5$ in.) was constructed and installed along the length of the room, 3 feet from and parallel to one wall. This floor provided a resonant walking surface with minimal dampening of impact sounds. Walkers wore the pair from the laboratory's set of broken-in, D-width Florsheim "Richfield" shoes that fit them best; the shoes ranged from 5 to 13 in U.S. men's whole sizes. These shoes had hard leather soles and hard synthetic heels. Audio recordings (16-bit, 44k sampling rate) were made using a Sony PCM-M1 digital audio recorder (DAT) and a Sony stereo microphone (Model ECM-MS957). ${ }^{2}$ The microphone was placed on a 4 -in. floor stand located 4 feet from, and centered relative to, the long dimension of the walking surface. Simultaneous with the audio recordings, video recordings were made with a Panasonic PVDV 402 digital video camera (mini DV). This stationary camera was mounted on a tripod 15 feet from and centered relative to the walking surface (directly behind the microphone), with the camera angle covering approximately 15 feet of total length on the walking surface.

Walkers were required to wear a pair of their own short pants, both to minimize extraneous sounds from the rubbing of pants leg material (a problem noted in pilot recordings) and to provide unobstructed video recordings of the lower legs, ankles, and shoes during stimulus generation. "Upright" posture was described to the walkers as walking with their backs straight and in a comfortable, relaxed manner from one end of the floor to the other, with gaze fixated on an eye-level point at the far end of the walking surface. A "stooped" posture was defined as shoulders and back bent forward, with gaze fixated approximately 2 feet in front of the participant's feet. After initial practice walking in each posture, the participants were recorded walking the length of the floor, always in the same direction, five times in both upright and stooped postures.

The audio recordings were directly input from the DAT to the audio channel of a computer (16-bit, 24,414 sampling rate) and were digitally edited to five steps. Because the initial and final steps of a movement are often atypical, these steps were removed from the digital stimuli. If more than five steps were still present, the initial or final step of the remaining steps with the subjectively determined lowest signal-to-noise ratio was iteratively removed until only five contiguous steps remained. If any of the resulting five-step walking sequences contained excessive background noise (e.g., from the room air handler) or irrelevant sounds (e.g., a squeak of shoe or floor, or the noise of pants material ${ }^{3}$ ), that stimulus was discarded. From the original 22 walkers, 15 yielded a minimum of two stimulus sequences in each posture; to maintain an equal number of stimuli across walkers and postures, only two recordings for each walker in each posture were used in the study. Finally, in order to have an equal number of walkers in each of two groups in the behavioral study, the stimuli from 1 walker were removed from the stimulus set, leaving a final set of 14 (6 male and 8 female) walkers, and thus a total of 56 five-step sound files.

Biomechanical analysis. Digital videotapes were edited into individual walking sequences that corresponded to each of the 56 sound files, and these were examined to provide preliminary analyses of the biomechanics of the walkers in the contrasted postures. During this analysis, laboratory staff often noted a number of biomechanical differences that seemed to relate (sometimes in hindsight) to the expected differences discussed earlier. Attempts to quantify these differences from the videos using rating scales yielded a reliability measure $[r(390)=.69, p<.05]$ that, although significant, was not considered adequate for the study. The biomechanical measurements were therefore limited to those that could be reliably quantified from still frames taken at three distinct, acoustically relevant points of a single intermediate stride of each video file. Defined in terms of the leg closest to the camera, the first frame was taken at toe-off, the point at which the toe of the planted foot broke contact with the walking surface. The second frame was taken when the swing leg was at the midpoint of the stride, and the third when the heel of the swing leg initiated contact with the floor. Two laboratory staff members, both with experience editing the video files, were trained in the standardized measurement procedures and then independently measured the defined set of biomechanical properties. For all of the measures, there was excellent agreement between the staff measurements $[r(390)=.99, p<.05]$. 
Back angle, the primary indicator of the success of the posture manipulation, was measured directly at the three stride positions using a protractor positioned on a transparency. This measure was the angle (in degrees) between a line through the hip and shoulder joints and a horizontal line parallel to the floor. Thus, perfect upright posture was $90^{\circ}$. Within postures, individual walkers exhibited very little variation in back angle across the three positions (largest $S E$ was $0.19^{\circ}$ ). Thus, the three values were averaged to produce a single measure of back angle for each of the 56 walking sequences. The remaining descriptive properties were computed from the geometry of measurements made using a ruler. Arm swing was the total horizontal distance traveled by the wrist during a single stride, and thus was the difference in wrist position between the first and last of the three still frames. The vertical height of the shoulder joint was measured at each of the stride positions, with the shoulder movement measure being the variability (standard deviation) in shoulder height across the three stride positions. On the basis of horizontal and vertical distance measurements, the angle between the nose and the shoulder joint relative to the horizontal plane was calculated, with the head movement measure being the variability in this angle across the three stride positions.

In addition to variability, the potentially important dynamic attributes of human gait are pace and the force vectors at shoe-floor contact. We lacked the sophisticated equipment required to dynamically measure pressure or force; attempting to map the shoe impact forces to sounds would be a separate study in its own right. Walker pace, however, is easily measured as the time between successive heel strikes. However, the television standard of 30 frames per second for our video cameras only allowed a precision of $33 \mathrm{msec}$. We therefore measured pace in terms of the time (number of digital sound samples) between the onsets of successive heel impact sounds (the interstep interval [ISI], with better than 1-msec precision). Although we treat ISI as our measure of pace, the true measure of pace was 1,000/ISI. For each five-step walking sequence, there were four ISI values.

\section{Results and Discussion}

The basic anthropometrical and biomechanical properties of the walkers are summarized in Table 1. The distribution of basic anthropometrical properties of our walkers was generally consistent with national norms (see, e.g., National Center for Health Statistics, 2003). The source contrast in this study was posture change, which is best indicated by a shallower back angle in the stooped posture. A direct indication of the success of the posture manipulation is the significance of this measure across postures $[t(26)=11.04, p<.0001$, one-tailed $]$.

The first explicit goal here was to provide exploratory within-posture analyses of the relationships between static and dynamic walker attributes, and then to compare these relationships across postures. Two principal components (PC) extractions with an orthogonal Varimax rotation and Kaiser normalization (using SPSS software) were performed on the four (posture-irrelevant) anthropometrical and the five (posture-specific) biomechanical attributes listed in Table 1. Here and elsewhere, an eigenvalue $>1$ was the criterion used to specify component inclusion.

For the upright posture, three components were extracted; all communality values for the variables were $\geq .63$, indicating well-defined variables in this solution. All variables met the criterion of at least a .5 loading on one of the three components in the rotated solution. Loadings of variables, communalities, and percentages of variance accounted for are shown in the left panel of Table 2; to aid interpretation, here and elsewhere loadings $<.5$ are not displayed. The first component, characterized by expected high loadings on height, weight, and shoe size, captures relationships to walker size; the inclusion of shoulder movement in this component seems reasonable, since shoulder displacement should be greater in larger walkers. In the second component, the presence of a loading for gender but not for physical size indicates that pace is related to gender rather than body size. The negative loading of arm swing reflects the need to counter the faster (more forceful) rotational movement of the lower body that is an aspect of the increased pace. Finally, the third-component loadings indicate a known relationship between back angle and upward vertical movement of the body, here exhibited by movement of the head (Broer, 1973).

In the stooped analysis, three components were also extracted, with all communality values $\geq .59$ and all variables meeting the component-loading criterion for inclusion in the rotated solution; the results are summarized in the right panel of Table 2. The first stooped-posture component includes the basic anthropometric size variables seen in the primary upright-posture component. Beyond gender, there now appears to be a dependency of pace on anthropometrical attributes of the walker. Other differences with the upright posture include shoulder movement now being related to back angle, as well as a relationship between arm swing and head movement. These changes in variable relationships seem consistent with the very broad biomechanical modifications that adapt to the change in center of gravity.

By themselves, changes in the patterns of shared variances for static and dynamic properties across postures tell us little about the utility of the individual biomechanical attributes in differentiating the posture manipulation. Independent-measures $t$ tests were used to identify significant (thus, systematic across walkers) changes in the biomechanical properties across postures. Because multiple tests were performed here and in later analyses, we controlled for familywise error rates by adopting an alpha level of .01. In the across-walker data, no biomechanical measure other than back angle (discussed above) differed significantly across postures. ${ }^{4}$ If walkers had adopted similar strategies in order to cope with the posture change, the lack of statistical separation across walkers for our other measures probably would be due to considerable variability in the anthropometrical and biometrical attributes of the walkers. To factor out across-walker variability from the evaluation of biomechanical posture differences, a separate set of within-walker analyses were performed using paired-samples $t$ tests. Not surprisingly, back angle continued to be highly significant $[t(13)=14.47, p<$ $.0001]$. The PC analyses indicated postural differences in the relationships among the biomechanical measures, and now that basic across-walker variability had been factored out, some of these variables were found to be significant - specifically, pace $[t(13)=5.14, p<.0001]$ and arm swing $[t(13)=2.93, p=.005]$. The lack of significance for head [t(13) $=1.57, p=.07]$ and shoulder $[t(13)=1.05, p=.16]$ movement may simply indicate that large biomechanical changes took place in the properties for some, but not all, walkers.

Probably other biomechanical changes did occur because of the manipulation of posture, but those changes 
Table 1

Means and Standard Errors for Anthropometrical and Biomechanical Properties

\begin{tabular}{|c|c|c|c|c|c|c|c|c|c|c|c|c|c|c|}
\hline \multirow[b]{4}{*}{ Walker } & & & & & \multicolumn{10}{|c|}{ Dynamic Biomechanical Properties } \\
\hline & \multicolumn{4}{|c|}{ Static Anthropometrical Properties } & \multirow{2}{*}{\multicolumn{2}{|c|}{$\begin{array}{l}\text { Back } \\
\text { Angle } \\
\text { (deg.) }\end{array}$}} & \multirow{2}{*}{\multicolumn{2}{|c|}{$\begin{array}{c}\text { Head } \\
\text { Mvmt } \\
(\sigma \text { deg. }) \\
\end{array}$}} & \multirow{2}{*}{\multicolumn{2}{|c|}{$\begin{array}{c}\text { Shoulder } \\
\text { Mvmt } \\
(\sigma \text { deg. }) \\
\end{array}$}} & \multirow{2}{*}{\multicolumn{2}{|c|}{$\begin{array}{c}\text { Arm } \\
\text { Swing } \\
\text { (in.) }\end{array}$}} & \multirow{2}{*}{\multicolumn{2}{|c|}{$\begin{array}{l}\text { Pace } \\
\text { (ISI) }\end{array}$}} \\
\hline & Height & Weight & Shoe Size & & & & & & & & & & & \\
\hline & (in.) & (lbs.) & (U.S. Men's) & Gender & $\mathrm{U}$ & $\mathrm{S}$ & $\mathrm{U}$ & $\mathrm{S}$ & $\mathrm{U}$ & $\mathrm{S}$ & $\mathrm{U}$ & $\mathrm{S}$ & $\mathrm{U}$ & $\mathrm{S}$ \\
\hline 1 & 64 & 117 & 5 & $\mathrm{~F}$ & 90 & 43 & 0.04 & 0.04 & 2.7 & 2.5 & 38.8 & 7.5 & 522 & 486 \\
\hline 2 & 74 & 156 & 11 & M & 89 & 48 & 0.03 & 0.04 & 3.0 & 3.0 & 12.5 & 18.0 & 667 & 588 \\
\hline 3 & 69 & 144 & 9 & $\mathrm{~F}$ & 88 & 51 & 0.03 & 0.13 & 2.8 & 4.0 & 13.9 & 6.5 & 662 & 644 \\
\hline 4 & 65 & 159 & 7 & $\mathrm{~F}$ & 89 & 33 & 0.08 & 0.04 & 2.5 & 0.6 & 28.8 & 14.9 & 586 & 568 \\
\hline 5 & 66 & 126 & 5 & $\mathrm{~F}$ & 84 & 35 & 0.01 & 0.08 & 0.8 & 0.5 & 8.5 & 1.4 & 546 & 501 \\
\hline 6 & 70 & 149 & 10 & M & 88 & 52 & 0.04 & 0.04 & 2.0 & 2.9 & 1.5 & 0.1 & 658 & 633 \\
\hline 7 & 69 & 136 & 9 & M & 85 & 46 & 0.04 & 0.01 & 1.7 & 1.4 & 24.0 & 34.5 & 574 & 560 \\
\hline 8 & 65 & 140 & 7 & $\mathrm{~F}$ & 89 & 37 & 0.04 & 0.03 & 0.2 & 0.6 & 3.0 & 8.3 & 606 & 568 \\
\hline 9 & 67 & 153 & 7 & $\mathrm{~F}$ & 90 & 61 & 0.04 & 0.04 & 3.1 & 0.4 & 23.5 & 6.0 & 552 & 554 \\
\hline 10 & 66 & 140 & 7 & $\mathrm{~F}$ & 87 & 53 & 0.01 & 0.05 & 1.5 & 2.2 & 53.8 & 32.9 & 541 & 497 \\
\hline 11 & 74 & 195 & 9 & $\mathrm{~F}$ & 92 & 41 & 0.06 & 0.07 & 3.1 & 1.8 & 31.0 & 5.1 & 659 & 643 \\
\hline 12 & 70 & 131 & 8 & M & 88 & 35 & 0.04 & 0.04 & 1.4 & 1.0 & 27.3 & 0.6 & 566 & 519 \\
\hline 13 & 72 & 186 & 10 & M & 84 & 31 & 0.01 & 0.09 & 3.6 & 1.3 & 17.4 & 9.8 & 601 & 569 \\
\hline 14 & 64 & 165 & 8 & M & 101 & 82 & 0.05 & 0.06 & 2.3 & 3.4 & 11.0 & 6.5 & 593 & 592 \\
\hline$M$ & 68 & 150 & 8.0 & - & 89 & 46 & 0.04 & 0.05 & 2.2 & 1.8 & 21.1 & 10.9 & 595 & 566 \\
\hline$S E$ & 0.9 & 5.8 & 0.5 & - & 1.2 & 3.7 & 0.01 & 0.01 & 0.3 & 0.3 & 3.9 & 2.9 & 13.2 & 13.9 \\
\hline
\end{tabular}

Note-ISI, interstep interval in milliseconds; U, upright; S, stooped.

were likely complex, were probably interrelated, and were not evident in any single variable we were able to measure reliably. The posture-related changes in the dynamic forces of impact during walking probably are also complex and interrelated. If this is the case, it is unlikely that simple, invariant cues exist that could indicate posture or posture change. Instead, posture contrasts will most likely be reflected only statistically for a subset of individual acoustic properties (e.g., pace), and possibly only probabilistically in the pattern of impact sound properties.

\section{ANALYSIS OF SOUND}

The logical progression of analyses brings us to the next corner of the information triangle, the sound produced. Beyond specifying the properties that characterized each walking sound, we first examined the broad, general relationships between the source event and the resulting sound structures within each posture. We then sought to determine the probability that each property (both separately and combined) could differentiate the posture contrast.

\section{Method}

The five steps in each of the 56 walking sequences contained distinct acoustic peaks corresponding to heel and initial sole impacts with the floor. Approximately $30 \%$ of the steps had a third impact sound, typically a second sole impact from the contact between the front of the foot (pivoting to toe-off) and floor. Because the rate of third impact sounds per step and also per walker was nearly identical across postures, third impact sounds will not be considered further. Across all walkers, the stimulus onset asynchrony (SOA) of impact sounds within each step was typically less than $80 \mathrm{msec}$, with some just over $40 \mathrm{msec}$. To obtain an optimum, yet standardized, signal length for fast Fourier transform (FFT) analyses, while also ensuring that each impact was largely devoid of contamination from the next impact sound, all impact sounds were edited to a length of $40 \mathrm{msec}$, beginning at the waveform zero-crossing immediately preceding the sharp rise in amplitude due to initial impact. Spectral properties for

Table 2

Factor Loadings (C1-C3), Communalities $\left(h^{2}\right)$, and

Percentages of Variance for Principal Components Extraction and Varimax Rotation on Walker Anthropometrics and Biomechanics

\begin{tabular}{|c|c|c|c|c|c|c|c|c|}
\hline & \multicolumn{4}{|c|}{ Upright } & \multicolumn{4}{|c|}{ Stooped } \\
\hline & $\mathrm{C} 1$ & $\mathrm{C} 2$ & $\mathrm{C} 3$ & $h^{2}$ & $\mathrm{C} 1$ & $\mathrm{C} 2$ & $\mathrm{C} 3$ & $h^{2}$ \\
\hline \multicolumn{9}{|l|}{ Anthropometrics } \\
\hline Weight & .79 & & & .75 & .70 & & & .67 \\
\hline Height & .72 & & & .85 & .91 & & & .67 \\
\hline Shoe size & .65 & .70 & & .92 & .94 & & & .75 \\
\hline Gender & & .74 & & .63 & .60 & .53 & & .75 \\
\hline \multicolumn{9}{|l|}{ Biomechanics } \\
\hline Arm swing & & -.84 & & .80 & & & -.81 & .82 \\
\hline Pace & & .76 & & .82 & & & & .98 \\
\hline Back angle & & & .87 & .76 & & .85 & & .59 \\
\hline Head movement & & & .84 & .71 & & & .78 & .65 \\
\hline Shoulder movement & .85 & & & .75 & & .86 & & .85 \\
\hline$\%$ Variance & 29.30 & 28.90 & 19.40 & & 35.20 & 22.10 & 17.20 & \\
\hline
\end{tabular}


the approximately 650 separate impact sounds were measured using FFT analyses (with Hamming window) implemented in MATLAB (The Mathworks, Inc.).

\section{Results and Discussion}

Spectral properties of sound. All impact sounds exhibited similar overall patterns of spectral distribution that can be broadly characterized as a skewed, inverted $\mathrm{V}$ shape. Specifically, below $100 \mathrm{~Hz}$, there was little (if any) difference from the background, followed by a rapid systematic rise in amplitude, up to the peak frequency that always was between 150 and $400 \mathrm{~Hz}$. This rise was then followed by a more gradual systematic fall in amplitude across the higher frequencies, reaching background level by, if not before, $3000 \mathrm{~Hz}$. Thus, the FFT analyses were limited to a range from 100 to $3000 \mathrm{~Hz}$. The measured spectral properties of the impact sounds were selected to capture the main aspects of this pattern, with one of these measures being peak frequency.

As indicators of timbre, spectral slope and spectral centroid were measured in both linear and log coordinate systems. Spectral slope was computed by a regression of amplitude as a function of frequency over the frequency range from the peak frequency to $3000 \mathrm{~Hz}$; for spectral slope (log), correlations between actual and predicted values ranged from .75 to .90 , indicating high goodness of fit. ${ }^{5}$ Also measured was the overall root-mean square amplitude, in decibels, in each of five adjacent, nonoverlapping frequency bands, starting with $100-500 \mathrm{~Hz}$ and proceeding through the four subsequent 500 -Hz-wide intervals. Of the temporal properties examined, we will report only the ISI between heel impacts, which (as noted in the Source Events section) is an indicator of pace. Table 3 provides a broad summary of the spectral properties (averaged across walkers), presented both separately for each posture and averaged across postures (a more detailed listing of the property measures for each walker is available on our Web page).

Sound with anthropometrics and biomechanics. The immediate goal was to examine the relationships between the measured spectral sound properties and the source properties of the walkers within each posture, accomplished here using PC analyses. Because the source properties were measured using interval (rather than ratio) scales, we used only the interval measures of spectral properties. PC extractions with an orthogonal Varimax rotation and Kaiser normalization (using SPSS) were performed. As in the source evaluation, separate posture-specific analyses were performed. For each posture, five components were extracted; all communality values were $\geq .52$, and all variables met the loading criterion for inclusion. Loadings of variables, communalities, and percentages of variance are shown in Table 4.

Although there should be consistency with the previous source analyses (Table 2), because total variance was now distributed across additional variables, there could be, and indeed were, changes in the component relationships for the source properties. For the upright posture, the primary component was essentially a combination of the first two components in the source analysis, with no significant contribution of any spectral property. All of the remaining components included at least one source and one sound property. The first of these (Component 2) seemed to show a relationship between the spectral central tendency of heel impact with shoulder movement. Component 3 reflected the same spectral central tendency, but now between sole impact and back angle. Component 4 seemed to combine anthropometrical properties that probably are related to body mass with spectral slope for both impact sounds. In general, it seems that different source attributes influence spectral properties of the sound at different stages of weight transfer, which makes sense in terms of the previous analysis of biomechanics.

With common anthropometrics but posture-altered biomechanics, the PC analyses for the stooped posture should show some similarities to, but also marked differences from, the upright PC analyses. The most striking difference was the addition of the heel impact sounds to the major source attributes in the primary component. This provided a statistical indication of the expected changes in manner and force of shoe placement. Component 2 only reflected sound properties, which contrasted with the upright posture, in which these same spectral distribution properties covaried with walker (thus, source) gender and weight. One implication was that some posture-related changes in biomechanics were reflected in the sounds

Table 3 Average Values Across Walkers for Spectral Analysis

\begin{tabular}{|c|c|c|c|c|c|c|c|}
\hline \multirow[b]{2}{*}{ Acoustic Property } & \multirow[b]{2}{*}{ Units } & \multicolumn{3}{|c|}{ Heel Impact } & \multicolumn{3}{|c|}{ Sole Impact } \\
\hline & & $\mathrm{U}$ & $\mathrm{S}$ & $M$ & $\mathrm{U}$ & $\mathrm{S}$ & $M$ \\
\hline \multicolumn{8}{|c|}{ Broad Spectral Indicators } \\
\hline Slope $(\log )$ & $\log (\mathrm{A} / \mathrm{Hz})$ & -3.7 & -3.8 & -3.7 & -4.0 & -3.8 & -3.9 \\
\hline Slope (linear) & $\mathrm{A} / \mathrm{kHz}$ & -0.058 & -0.128 & -0.093 & -0.028 & -0.055 & -0.042 \\
\hline Centroid (log) & $\mathrm{Hz}$ & 236.4 & 248.6 & 242.5 & 294.0 & 305.9 & 299.9 \\
\hline Centroid (linear) & $\mathrm{Hz}$ & 254.3 & 268.0 & 261.2 & 336.4 & 353.0 & 344.7 \\
\hline Peak frequency & $\mathrm{Hz}$ & 220.7 & 231.1 & 225.9 & 244.9 & 255.3 & 250.1 \\
\hline \multicolumn{8}{|c|}{ Frequency Band Amplitudes } \\
\hline $100-500$ & $\mathrm{~dB}$ & -20.3 & -19.6 & -20.0 & -10.9 & -11.4 & -11.1 \\
\hline $500-1000$ & $\mathrm{~dB}$ & -29.0 & -28.8 & -28.9 & -20.4 & -19.4 & -19.9 \\
\hline $1000-1500$ & $\mathrm{~dB}$ & -32.8 & -33.2 & -33.0 & -26.8 & -25.6 & -26.2 \\
\hline $1500-2000$ & $\mathrm{~dB}$ & -36.8 & -36.9 & -36.9 & -32.2 & -31.4 & -31.8 \\
\hline $2000-2500$ & $\mathrm{~dB}$ & -39.1 & -39.3 & -39.2 & -36.0 & -34.8 & -35.4 \\
\hline
\end{tabular}


Table 4

Factor Loadings (C1-C5), Communalities $\left(h^{2}\right)$, and Percentages of Variance for Principal Components Extraction and Varimax Rotation on Walker Anthropometrics, Biomechanics, and Spectral Properties

\begin{tabular}{|c|c|c|c|c|c|c|c|c|c|c|c|c|}
\hline & \multicolumn{6}{|c|}{ Upright } & \multicolumn{6}{|c|}{ Stooped } \\
\hline & $\mathrm{C} 1$ & $\mathrm{C} 2$ & $\mathrm{C} 3$ & $\mathrm{C} 4$ & $\mathrm{C} 5$ & $h^{2}$ & $\mathrm{C} 1$ & $\mathrm{C} 2$ & $\mathrm{C} 3$ & $\mathrm{C} 4$ & $\mathrm{C} 5$ & $h^{2}$ \\
\hline \multicolumn{13}{|l|}{ Anthropometrics } \\
\hline Height & .95 & & & & & .93 & .66 & & & & & .86 \\
\hline Shoe size & .91 & & & & & .92 & .83 & & & & & .96 \\
\hline Gender & .61 & & & -.52 & & .86 & .66 & & & & & .87 \\
\hline Weight & .59 & & & -.57 & & .83 & .65 & & & .63 & & .76 \\
\hline \multicolumn{13}{|l|}{ Biomechanics } \\
\hline Pace & .76 & & & & & .85 & .82 & & & & & .83 \\
\hline Shoulder movement & & .62 & & & & .79 & .65 & & & & & .85 \\
\hline Back angle & & & .79 & & & .93 & & & & .69 & & .77 \\
\hline Arm swing & & & & & -.90 & .94 & & & & & .64 & .76 \\
\hline Head movement & & & & & & .52 & & & .68 & & & .72 \\
\hline \multicolumn{13}{|l|}{ Spectral Properties } \\
\hline Peak frequency (heel) & & .94 & & & & .91 & .76 & & & & & .65 \\
\hline Spectral centroid (heel) & & .86 & & & & .84 & .68 & & & & & .95 \\
\hline Peak frequency (sole) & & & .92 & & & .92 & & -.58 & & & & .94 \\
\hline Spectral centroid (sole) & & & .73 & & & .95 & & .91 & & & & .82 \\
\hline Spectral slope (heel) & & & & .84 & & .81 & & & .80 & & & .94 \\
\hline Spectral slope (sole) & & & & .57 & .71 & .96 & & .81 & & & & .80 \\
\hline$\%$ Variance & 25.51 & 17.92 & 15.48 & 15.45 & 11.97 & & 22.57 & 17.65 & 15.80 & 14.60 & 12.58 & \\
\hline
\end{tabular}

produced, with the apparent decoupling of these sound attributes from anthropometrics probably indicating large individual differences in the nature of the biomechanical strategies adopted to compensate for the altered center of gravity. Component 3 also indicated a change in the relationship between biomechanics and sound, now with spectral centroid of heel impact sounds related to head, rather than to shoulder, movement. The remaining components strictly included source attributes.

Statistical differentiation of source events. The relationships between source and sound were clearly altered by the posture change, but differences in the within-posture patterns of covariation did not provide any indication of the degree to which sound properties consistently differed across postures. Traditional analyses using standard inferential statistics provide an initial indication of the average (across-walker) posture-diagnostic capabilities of the acoustic properties. None of the examined properties listed in Table 4 were significantly posture diagnostic. ${ }^{6}$ Since the mapping of the complex source dynamics to sounds is also complex, it is not surprising to find a lack of significance in standard inferential statistics for these simple measures of sound. The overall failures to reject the null hypotheses (the purpose of inferential statistics) do not necessarily indicate the absence of posture-diagnostic information in some of the properties for subsets of the walkers; we will later consider this possibility.

Although the properties of the discrete impact sounds may not have been posture diagnostic across all walkers, it is possible that posture contrast could be conveyed systematically by some within-step pattern of acoustic properties. In examining possible configural properties, we sought to identify posture-related differences that were both statistically important and probably perceptible to human listeners. Because we did not find any properties that met these criteria, we provide here only a very brief summary of a few of the examined patterns. There was a within-step upward timbre shift (peak frequency and spectral centroid) between heel and sole impact sounds that was slightly larger in the stooped posture, but the average difference across postures was only $15-17 \mathrm{~Hz}$. Next, since walker pace was typically faster (i.e., ISI was smaller) in the stooped posture, one might expect more rapid foot placement (heel-sole impact timing), and possibly a higher incidence of an extra impact sound, but there was no evidence of either difference across postures. Finally, for all frequency bands analyzed, sole impacts were usually more intense than heel impacts, with a small $(1-2 \mathrm{~dB})$ average difference across postures, but also a fair amount of variability. This within-step amplitude change was large and posture diagnostic, but only for very few walkers (e.g., a 9-dB low-frequency contrast for Walker 7).

Signal detection modeling. Our goal was to evaluate the posture-diagnostic capabilities of the acoustic properties. This required the use of descriptive, rather than inferential, statistics, which were first implemented across walkers, and then for individual walkers. We began by using two implementations of ideal-observer modeling. Such models make decisions on the basis of statistical evaluations of available evidence utilizing a Bayesian, or likelihood ratio, decision variable (Wald, 1955). Our implementation was based on the common Gaussian equalvariance model of signal detection theory (SDT; Pastore, Crawley, Berens, \& Skelly, 2003; Tanner, 1960, 1961). For each acoustic property, we analyzed the probability of accurately differentiating posture contrast by computing $d^{\prime}$ and then converting the value to $p(\mathrm{c})_{\max }$ (Macmillan \& Creelman, 1991, 2005). This was done first for $d^{\prime}$ computed for each acoustic property from the across-walker mean and variance. ${ }^{7}$ From our theoretical and actual analyses of the biomechanics of the posture change, we 
expected high across-walker variability that would probably severely handicap simple, broad analyses. The majority of the acoustic properties differentiated posture at near-chance levels (the upper portion of Table 5 provides a complete summary of this analysis). However, a few acoustic properties were somewhat better than chance, with best performance for the (linear) spectral slope of both heel and sole impact sounds $\left[p(\mathrm{c})_{\max } \approx .65\right]$ and pace $\left[p(c)_{\max }=.63\right]$. Even if we could assume that all of the sound properties were orthogonal, the combined diagnostic potential of all of the across-walker statistics would not be very good [e.g., $p(\mathrm{c})_{\max }=.70$ for the combined spectral slope of impacts].

Because the nature and range of the possible consequences of the posture change depended on the anthropometrical and biomechanical characteristics of each individual walker, the acoustic manifestations of posture change were expected to differ across walkers. The second ideal-observer modeling analysis was thus based on the across-step statistics of each acoustic property for each individual walker. This within-walker analysis eliminated the across-walker variability, but added across-step variability for each walker in each posture. ${ }^{8}$ Although the average diagnostic potential of many of the acoustic properties was still close to chance, it was much higher for some of the properties. Specifically, for pace and (linear) spectral slope, the most diagnostic properties in the across-walker average accuracy, the results were now approximately .76. In addition, there was better diagnostic potential for the spectral centroid of heel impact sounds $\left[p(\mathrm{c})_{\max }=\right.$ .73] and the amplitude of sole impact sounds $\left[p(\mathrm{c})_{\max } \approx\right.$ $.63]$ in some of the frequency bands (the lower portion of Table 5 provides a summary of the across-walker averages from this analysis). Having identified somewhat better posture-diagnostic capabilities for several acoustic properties, we could now begin to look at the capabilities of the combined acoustic properties.

If we made the questionable assumption that the sound properties were orthogonal, the combination of pace and the heel spectral centroid properties would improve the posture-diagnostic accuracy to approximately .82, which is quite good. The PC analysis of the source event found a typical grouping of pace with height, weight, and shoe size, and the spectral distribution of energy should, at least in part, reflect both body and shoe size. If pace and spectral centroid were partially correlated, the joint posture- predictive ability of these sound properties would become less than .82. Our analysis of acoustic properties for individual walkers, however, did provide some indication of the distribution of the degree to which specific acoustic properties, both separately and together, reflected the posture change for the individual walkers. Pace was most diagnostic for Walker $1\left[p(c)_{\max }=.91\right]$ and next best for Walker $10\left[p(\mathrm{c})_{\max }=.86\right]$. Pace was only moderately posture diagnostic for most of the other walkers, but it was approximately chance for Walkers 9 and 14 . For those walkers specifically identified here, (linear) spectral slope for heel impact was excellent for Walker $10\left[p(\mathrm{c})_{\max }>\right.$ .95] but much lower for Walkers 1,9 , and $14\left[p(\mathrm{c})_{\max } \approx\right.$ .66]. Again, with the strong (but questionable) assumption that these two variables were orthogonal, the use of both properties would result in .98 posture identification accuracy for Walker 10, but in essentially no improvement (.92) for Walker 1.

The modeling we describe provided a much better understanding of the relationships between source and sound properties than was possible from broad statistical analyses, but, for several reasons, we reached a limit on the information a relatively straightforward SDT-based modeling could provide about the present natural source-event contrast. SDT modeling requires a reasonable estimate of variability, but the variability in some of our acoustic property measures reflected the (largely posture-irrelevant) change in distance as the walker approached, passed, and then moved away from the recording microphone. In addition, estimating the statistical diagnostic ability of combinations of acoustic properties required either a questionable assumption of independence or actual estimates of the correlation between the acoustic properties.

Property-based differencing model with reliability criterion. Although it is possible to develop likelihoodbased ideal-observer models with dynamic property weights that change on the basis of the likelihood ratio for individual properties (e.g., weights proportional to absolute value of log likelihood ratio), the nature of the needed assumptions and the level of sophistication in such modeling are well beyond the scope of the present, already very complex, project. We could, however, easily implement a relatively simple version of this strategy that meets the immediate goals of our project with simple models that do not rely on strong assumptions about the underlying distributions of or the relationships between the stimulus

Table 5

Signal Detection Theory: $\boldsymbol{p}(\mathrm{c})_{\max }$

\begin{tabular}{|c|c|c|c|c|c|c|c|c|c|c|c|}
\hline & \multirow[b]{3}{*}{$\begin{array}{l}\text { Pace } \\
\text { (ISI) }\end{array}$} & \multicolumn{5}{|c|}{ Broad Spectral Indicators } & \multirow{2}{*}{\multicolumn{5}{|c|}{ Frequency Band Amplitude }} \\
\hline & & & Slope & & Centroid & Peak & & & & & \\
\hline & & $\begin{array}{c}\text { Slope } \\
{[\log (\mathrm{A} / \mathrm{Hz})]}\end{array}$ & $\begin{array}{l}\text { (linear; } \\
\mathrm{A} / \mathrm{kHz} \text { ) }\end{array}$ & $\begin{array}{l}\text { Centroid } \\
(\log ; \mathrm{Hz})\end{array}$ & $\begin{array}{c}\text { (linear; } \\
\mathrm{Hz} \text { ) }\end{array}$ & $\begin{array}{c}\text { Frequency } \\
(\mathrm{Hz})\end{array}$ & $\begin{array}{c}100- \\
500\end{array}$ & $\begin{array}{l}500- \\
1000 \\
\end{array}$ & $\begin{array}{c}1000- \\
1500 \\
\end{array}$ & $\begin{array}{c}1500- \\
2000\end{array}$ & $\begin{array}{c}2000- \\
2500 \\
\end{array}$ \\
\hline \multicolumn{12}{|l|}{ Across Walkers } \\
\hline Heel strike & .63 & .54 & .66 & .61 & .60 & .59 & .54 & .51 & .52 & .50 & .51 \\
\hline Sole strike & & .60 & .65 & .55 & .56 & .53 & .53 & .56 & .57 & .55 & .58 \\
\hline \multicolumn{12}{|l|}{ Within Walkers } \\
\hline Heel strike & .76 & .53 & .78 & .73 & .68 & .66 & .57 & .53 & .51 & .50 & .50 \\
\hline Sole strike & & .62 & .76 & .43 & .43 & .45 & .47 & .60 & .64 & .59 & .63 \\
\hline
\end{tabular}

Note-ISI, interstep interval in milliseconds. 
property values. First, we implemented models for individual acoustic properties, and then we generalized the strategy to modeling pairs of properties, thus providing, within the same modeling framework, estimates of the posture-predictive information available for each property and the gain in accuracy from combining each of the bestperforming properties with every other property.

Single-property differencing models. Our propertybased two-alternative forced choice (2AFC) differencing models were adapted from classic high-threshold models (e.g., Luce, 1963; Macmillan \& Creelman, 1991, 2005). A 2AFC task, which tends to result in minimal bias, is effectively modeled by having the theoretical observer make decisions on the basis of differences in an evaluated property of the stimuli presented on a given trial (Green \& Swets, 1966; Macmillan \& Creelman, 1991, 2005). Our property-based differencing models (implemented in MATLAB) essentially operated in this fashion. The model for a given acoustic property compared, for each walker, the average value (across the five-step walking sequence) for the four possible across-posture pairings with the decision for each pairing based on the magnitude and direction of difference in value; the direction of difference in each case was identical to that for the property in the SDT models. The magnitude of difference was treated as a measure of reliability, and the "threshold" (the only free parameter) was a criterion for the reliability of evidence from the given measured acoustic property. If the magnitude of difference was greater than the reliability criterion, we used the difference to make a decision that, on the basis of the actual sources, was either correct or incorrect (i.e., each of the four decisions added, respectively, either .25 or .00 to total accuracy for the walker). In the simple, single- property models, a magnitude of difference less than the reliability criterion resulted in a guess that was correct on half of the trials (i.e., added .125 to total accuracy for the walker). The result for each walker measured the accuracy achievable from the given acoustic property as a function of reliability criterion, with total accuracy simply the average accuracy across walkers. Table 6 reports the maximum average across-walker accuracy across all possible reliability criteria.

The shape of the distribution patterns of average accuracy across most of the broad spectral and temporal properties closely matched the distribution from the within-walker SDT models $[r(8)=.93, p<.05]$, indicating that the two types of models probably reflect the same underlying information. However, there was a poor correlation between the two models in the distributions of accuracy across spectral band amplitude $[r(8)=-.11, p>.05]$. This low correlation is not surprising, since many of the predictions, especially from the SDT models, are at or near chance levels. This lower predicted accuracy from the SDT models may largely reflect the posture-irrelevant variability in amplitude across steps as a function of distance between the moving walker and the stationary microphone (contributing to the denominator in computing $d^{\prime}$ ); across-step variability is not a differencing model parameter. For the differencing models, only the low-frequency $(100-500 \mathrm{~Hz})$ band exhibited reasonably high accuracy, and it did so for both heel and sole impact sounds. Thus, the single-property differencing models not only reflected the same underlying relationships between sound properties and posture indicated in the within-walker SDT models, but also indicated a higher predictive value, now with pace (.87) and amplitude in the $100-$ to $500-\mathrm{Hz}$ range for sole impact (.86) being approxi-

Table 6

Property-Based Differencing Model by Walker

\begin{tabular}{|c|c|c|c|c|c|c|c|c|c|c|c|c|c|c|c|c|}
\hline & \multirow{2}{*}{$\begin{array}{l}\text { Impact } \\
\text { Sound }\end{array}$} & \multicolumn{14}{|c|}{ Walker } & \multirow[b]{2}{*}{$M$} \\
\hline & & 1 & 2 & 3 & 4 & 5 & 6 & 7 & 8 & 9 & 10 & 11 & 12 & 13 & 14 & \\
\hline Pace & & 1.00 & 1.00 & .75 & 1.00 & 1.00 & .88 & 1.00 & 1.00 & .50 & 1.00 & .50 & 1.00 & 1.00 & .50 & .87 \\
\hline \multicolumn{17}{|l|}{ Broad Spectral Indicator } \\
\hline \multirow[t]{2}{*}{ Linear spectral slope } & Heel & .50 & .75 & 1.00 & 1.00 & .50 & 1.00 & 1.00 & .75 & .88 & 1.00 & .75 & 1.00 & 1.00 & .50 & .83 \\
\hline & Sole & .50 & .00 & .88 & 1.00 & 1.00 & 1.00 & .88 & .88 & .75 & 1.00 & .75 & 1.00 & 1.00 & 1.00 & .83 \\
\hline \multirow[t]{2}{*}{ Log spectral slope } & Heel & .50 & .75 & .50 & 1.00 & .38 & .38 & .75 & .63 & .75 & .50 & .50 & .75 & .50 & .50 & .60 \\
\hline & Sole & 1.00 & 1.00 & 1.00 & .25 & .00 & .50 & 1.00 & 1.00 & .50 & 1.00 & .50 & 1.00 & .25 & .75 & .70 \\
\hline \multirow[t]{2}{*}{ Linear centroid } & Heel & .50 & .00 & .63 & .25 & 1.00 & 1.00 & 1.00 & .25 & 1.00 & 1.00 & 1.00 & 1.00 & .88 & .63 & .72 \\
\hline & Sole & .63 & .50 & .63 & .63 & .75 & 1.00 & .00 & .63 & .50 & .63 & .50 & .50 & .88 & .75 & .61 \\
\hline \multirow[t]{2}{*}{ Log centroid } & Heel & .38 & .25 & .63 & 1.00 & 1.00 & 1.00 & 1.00 & .38 & 1.00 & 1.00 & 1.00 & 1.00 & .88 & .75 & .81 \\
\hline & Sole & .75 & .50 & .50 & .75 & .63 & 1.00 & .00 & .50 & .50 & .50 & .75 & .50 & .75 & .75 & .60 \\
\hline \multirow[t]{2}{*}{ Peak frequency } & Heel & .25 & 1.00 & .50 & .88 & 1.00 & .50 & 1.00 & 1.00 & .63 & .25 & .63 & 1.00 & .50 & .25 & .67 \\
\hline & Sole & .50 & .25 & .75 & .88 & .63 & .75 & .75 & .50 & .75 & .13 & 1.00 & .38 & .63 & .50 & .60 \\
\hline \multicolumn{17}{|c|}{ Frequency Band Amplitude } \\
\hline \multirow[t]{2}{*}{$100-500$} & Heel & .25 & 1.00 & 1.00 & 1.00 & .00 & 1.00 & 1.00 & 1.00 & 1.00 & 1.00 & 1.00 & 1.00 & 1.00 & .50 & .84 \\
\hline & Sole & .50 & .00 & 1.00 & 1.00 & 1.00 & 1.00 & 1.00 & 1.00 & .75 & 1.00 & .75 & 1.00 & 1.00 & 1.00 & .86 \\
\hline \multirow[t]{2}{*}{$500-1000$} & Heel & .63 & .00 & .50 & .38 & 1.00 & 1.00 & 1.00 & .25 & .38 & .75 & .63 & .88 & .75 & .75 & .64 \\
\hline & Sole & .50 & .38 & .50 & .50 & .50 & .50 & .25 & .50 & .38 & .50 & .50 & .50 & .88 & .50 & .49 \\
\hline \multirow[t]{2}{*}{$1000-1500$} & Heel & .75 & .00 & .00 & .00 & 1.00 & 1.00 & 1.00 & .00 & .00 & .50 & 1.00 & .50 & 1.00 & 1.00 & .55 \\
\hline & Sole & .50 & .50 & .75 & .75 & 1.00 & 1.00 & .00 & 1.00 & .50 & 1.00 & .25 & .50 & .75 & 1.00 & .68 \\
\hline \multirow[t]{2}{*}{$1500-2000$} & Heel & .75 & .00 & .25 & .00 & 1.00 & 1.00 & .75 & .50 & .00 & .50 & .50 & .50 & 1.00 & .75 & .54 \\
\hline & Sole & .50 & .50 & .75 & .00 & 1.00 & 1.00 & .50 & 1.00 & .50 & 1.00 & .25 & .75 & .75 & 1.00 & .68 \\
\hline \multirow[t]{2}{*}{$2000-2500$} & Heel & .75 & .00 & .00 & .00 & 1.00 & 1.00 & 1.00 & .25 & .00 & .25 & 1.00 & .50 & 1.00 & 1.00 & .55 \\
\hline & Sole & .50 & .50 & .75 & .00 & .50 & 1.00 & .50 & 1.00 & .50 & .75 & .25 & 1.00 & .75 & .75 & .63 \\
\hline
\end{tabular}


mately equally predictive, and spectral slope (linear) of the heel impact (.83) only slightly lower.

Hierarchical differencing models. Implementation of any two-property model requires that assumptions be made about the correlation between and the scaling of variable values across properties, as well as about the nature of the decision process. For example, our SDT modeling implicitly assumed that the physical measurements for each property were appropriately scaled in terms of the variability of the measurements. Although the SDT modeling approach may have been reasonable for evaluation of the individual spectral and temporal properties, unless the scaling and correlation assumptions were empirically validated (Macmillan \& Kingston, 1995), the need for simplifying assumptions about these factors would be a major limitation for using this approach to implement models that evaluate the combined properties.

The hierarchical model included primary and secondary properties, representing two stages of evaluation. The first stage was essentially the single-property model for the primary property, making a correct versus incorrect decision when the reliability criterion was exceeded. The one modification to the single-property model for this initial stage occurred when the criterion was not exceeded; rather than guessing, the model evaluated the second property. This second stage was fully equivalent to the single-property model for the secondary property, making a correct versus incorrect decision when the relevant criterion was exceeded, and otherwise guessing. The model thus had two free parameters - specifically, the reliability criteria for each of the two properties. With the model making discrete within-property decisions, scaling across properties was unnecessary. Because the secondary property was evaluated only when the primary property information was weak (i.e., unreliable), accuracy could improve only when the secondary property provided valid diagnostic information not already available in the primary property. As a result, if the across-posture differences for a pair of acoustic measures covaried in the same way across walkers, accuracy would not be better for the hierarchical model than for the single-property model. In contrast, if anthropometrics or biomechanics resulted in large across-posture differences in one acoustic property for some walkers and in the alternative property for other walkers, accuracy would improve in the hierarchical model.

When implementing the hierarchical models, we compared a large range of criteria for each set of acoustic properties. Because pace, (linear) spectral slope of the heel impact, and amplitude in the $100-500 \mathrm{~Hz}$ range of sole impact produced the highest accuracy in the simple models, the hierarchical models were based on one of these as the primary property, with the second source of information sampled exhaustively from the acoustic properties listed in Table 6. The highest predicted accuracy for the hierarchical two-property models was obtained when the second property was also one of these three acoustic properties (thus, they are the only values reported). The finding of higher accuracy for the hierarchical models is evidence that changes in acoustic properties varied across walkers.

Table 7 provides the optimum predictions of the hierarchical models. What is somewhat surprising is that combinations of the acoustic properties produced a different pattern of results depending on the order. Any hierarchical model involving pace resulted in higher predicted accuracy when pace was the initial property; the model that began with pace and then checked the linear spectral slope of the heel impact increased maximum accuracy from .87 (single-property pace model) to .97 , whereas the model that reversed this order of properties increased maximum accuracy only to .92 . This asymmetry can be understood by considering the single-property and hierarchical models. When the single-property model evaluates pace, the information is largely either strong and correct or weak, with errors primarily reflecting incorrect guesses. If slope sometimes exhibits large but posture-reversed differences, equivalent single-property error rates reflect greater incorrect information and less guessing. Guessing in the single-property model translates into a first-stage opportunity for improvement from second-stage information. Because, for the hierarchical model, pace offers more opportunity for second-stage improvement than does slope, pace seems to change in a more similar fashion across walkers. A similar pattern, but with greater asymmetry, is exhibited in a comparison of pace and amplitude.

If human listeners are as sensitive as the instruments used to measure the sound properties and are able to use the relevant information (and to ignore the irrelevant information) with an efficiency and in a manner similar to our models, then human listeners should be able to exhibit nearly perfect performance. Our modeling also indicates that several listening strategies can achieve nearly equivalent performance. Thus, similar levels of high accuracy may not be indicative of similar listening strategies.

\section{HUMAN PERCEPTION OF THE SOURCE EVENT}

The study has now developed a good conceptualization of the fuzzy nature of the source events being studied and their relationships to sound. The final aspect of the study

Table 7

Abbreviated Hierarchical Property-Based Differencing Model

\begin{tabular}{lllrr}
\hline \multicolumn{1}{c}{ Primary Property } & Location & \multicolumn{1}{c}{ Secondary Property } & Location & $p(\mathrm{c})_{\text {max }}$ \\
\hline Pace & & Linear spectral slope of heel impact & Heel & .97 \\
Pace & & Amplitude (100-500) of sole impact & Sole & .98 \\
Linear spectral slope of heel impact & Heel & Pace & .92 \\
Linear spectral slope of heel impact & Heel & Amplitude (100-500) of sole impact & Sole & .95 \\
Amplitude (100-500) of sole impact & Sole & Pace & Heel & .93 \\
Amplitude (100-500) of sole impact & Sole & Linear spectral slope of heel impact & He \\
\hline
\end{tabular}


was to evaluate the ability of listeners to differentiate the contrasted walking source event of posture, and then to begin to evaluate the decision strategies of the listeners. From our source and sound analyses, it seemed clear that the task of our listeners, and the challenges for this phase of the study, could best be accomplished with posture decisions made for within-walker sound comparisons. Because this task would eliminate within (but not across) trials those acoustic property differences that reflect the characteristics of walkers that are orthogonal to posture contrast, it should make it somewhat easier for listeners to identify likely hypotheses, and to confirm or reject expectations, about posture-related differences in the sounds. More importantly for achieving our study goals, the across-walker differences in the posture-diagnostic potential of the various sound properties meant that the across-walker pattern of posture judgment accuracy exhibited by an individual listener should depend on the sound property (or properties) used by the listener in making the posture judgment.

\section{Method}

Participants. Sixty-eight students from the Binghamton University psychology department subject pool volunteered to participate in the study as fulfillment of a partial requirement for an undergraduate psychology course.

Stimuli. The 56 acoustic stimuli were the four (two upright and two in stooped position) edited five-step walking sequences from each of the 14 walkers. Early in the study, the walkers had been sorted into two groups, then arbitrarily labeled 1-7 (Stimulus Group A) and 8-14 (Stimulus Group B). To the degree possible, the two groups had equivalent distributions across the anthropometrical properties of height, weight, shoe size, and gender. On the basis of walker group, we defined two stimulus sets (A and B), each containing the four possible across-posture within-walker pairings of the two upright and two stooped walking sequences; each stimulus set thus had four unique pairs of walking sequences from 7 walkers. Stimuli were presented to listeners binaurally over Sennheiser HD 265 headphones at an average level of approximately $65 \mathrm{~dB}(\mathrm{C})$.

Procedure. Listener ability to judge posture was evaluated by a 2 AFC task with the two stimuli separated by a 500 -msec interstimulus interval. Accuracy in a $2 \mathrm{AFC}$ task tends to be empirically (Macmillan \& Creelman, 2005), if not theoretically (Green \& Swets, 1966), bias-free. On each trial, one pair of posture-contrasted walking sequences, generated by one walker, was presented. The listeners' task was to identify which sequence had been generated in the upright posture, responding with the " 1 " (first sequence upright) or "2" (second sequence upright) keys on the computer keyboard. Feedback was provided immediately after each response. Each listener participated in three experimental blocks, beginning with either Stimulus Set A (Listener Group A) or B (Listener Group B).

The initial (practice) block of trials consisted of a basic stimulus set with the 28 unique posture comparisons. In each of the remaining blocks, the basic stimulus set was presented twice, and thus consisted of 56 comparisons. On each trial, the computer program randomly selected without replacement from the block of possible stimulus comparisons, and then randomly determined stimulus order. The stimulus set from the practice block also was used in the second block of trials, which thus measured experienced performance. The final block used the other stimulus set, and thus measured generalization performance.

Before both the practice and the experienced blocks, but not before the generalization block, listeners were familiarized with the source event in two different ways. First, they viewed a video (with sound) of a research assistant walking in each posture; stimuli from this walker were not used in the experiment. The listener then was asked to walk the length of the carpeted laboratory in each of the two postures, thus providing the listener with direct experience of the biomechanical consequences of the contrasting postures.

After completion of the final (generalization) block, every listener was asked to describe his or her judgment strategy. The initial listeners were tape-recorded as they answered the scripted, open-ended question, "What kind of strategy did you use to tell the difference between stooped and upright?" A secretary transcribed these reports, which were checked and corrected by the laboratory staff. Listeners consistently reported making judgments based on perceived acoustic properties, rather than on either biomechanical source properties or source-related acoustic structure; the introspective reports thus were not explicitly consistent with the basic premise of direct perception. After adjusting for variability in vocabulary, the reports could be characterized as identifying a sampling of a limited set of acoustic properties that differed somewhat across listeners.

To avoid the lengthy transcription process and to better organize the self-report data, all later listeners (approximately half) were given a questionnaire that reflected the six most typical of the identified acoustic properties: pace, timbre, ${ }^{9}$ presence or absence of a third impact within individual steps, amplitude or loudness, ability to identify individual walkers, and the presence of specific or extraneous sounds, as well as a seventh item ("other") that allowed specification of any property not listed. Participants used a 5-point scale to identify the frequency of use of each of the listed stimulus properties.

\section{Results and Discussion}

The goal of the behavioral segment of the present study was to identify what information was used by listeners to successfully perform the posture judgment task. Because participants from a subject pool bring to an experiment a wide range of motivations and listening abilities, both qualities that contribute to performance, there should have been, and was, a wide range of accuracy across listeners. The average probability of correct responses across all three blocks of trials was .69 (range .38-.92). Assuming a high-threshold model, an average accuracy of .69 would be achieved by having valid information on slightly less than $40 \%$ of the trials and guessing correctly $(p=.5)$ on half of the remaining trials..$^{10}$ Although it is possible that some aspect of the poor performance was due to an auditoryinformation-to-source-posture mapping that resulted in below-chance performance for either some or all walkers, we cannot easily differentiate this from other causes of low performance. Thus, to better analyze listener decision processes, we focused on the listeners who exhibited an accuracy of at least .75 in either the experienced or the generalization block. From the original set of 68 listeners, 34 met this criterion (16 in Group A, 18 in Group B); unless otherwise indicated, only data from these better-performing listeners are reported.

Average across-walker pattern of accuracy. Table 8 summarizes for each of the three blocks of trials the average across-walker accuracy for each listener group. A mixed-design ANOVA was performed on the listener data with one within-subjects factor (block) and one betweensubjects factor (listener group). The ANOVA revealed a significant main effect of block $[F(2,31)=4.148, p=$ $.025]$. Post hoc tests indicated that across listener groups, no significant difference was present between the experienced performance and generalization blocks $[t(33)=$ $0.70, p=.49]$. However, there was a significant difference between practice and generalization blocks $[t(33)=$ 
Table 8

Probability Correct for Better Listeners

\begin{tabular}{lccccccc}
\hline \multirow{2}{*}{\multicolumn{1}{c}{ Type }} & \multicolumn{3}{c}{ Group A } & & \multicolumn{3}{c}{ Group B } \\
\cline { 2 - 3 } \cline { 7 - 8 } \cline { 6 - 8 } & Walkers & $M$ & $S E$ & & Walkers & $M$ & $S E$ \\
\hline Practice & $1-7$ & .72 & .04 & & $8-14$ & .78 & .03 \\
Experienced & $1-7$ & .84 & .02 & & $8-14$ & .80 & .01 \\
Generalization & $8-14$ & .82 & .02 & & $1-7$ & .83 & .02 \\
\hline
\end{tabular}

$2.57, p=.015]$, which is clearly based on the change for Group A.

The average group accuracy for each walker is plotted in Figure 1. In the ANOVA, there was no main effect of listener group $[F(1,32)=0.44, p=.514]$, and also no interaction between listener group and block $[F(2,32)=2.82$, $p=.07]$. Indeed, Figure 1 does exhibit a consistent pattern of performance across groups, with a correlation between them $[r(12)=.81, p<.05]$, but is the trend important? By having intentionally avoided the high degree of stimulus controls typical in most studies, we have not imposed severe constraints on either the relevant information available in the stimuli or the decision strategies that could lead to at least moderate accuracy. Unless listeners adopted highly similar source decisions, there should have been considerable variability, not uniformity, in listener performance across walkers. This variability is evident in two patterns of results: the distribution of across-listener accuracies for individual walkers, and the distribution of individual listeners' accuracies across walkers. For every walker, at least 1 listener was perfect at identifying posture, and sometimes as many as 20 were; only 5 walkers had at least 1 listener, and never more than 2 , who exhibited below-chance accuracy. As for individual listeners, 32 achieved perfect accuracy for a median of 5 walkers, and several of these listeners were always able to identify posture for more than half ( 8 or 9 ), but never for all 14 , of the walkers.
Listener self-reports also indicated considerable differences in the acoustic information that individuals believed they were using to make the posture judgments. The full set of 68 listeners was partitioned into three groups on the basis of overall accuracy. Those with the highest accuracy typically reported the frequent ("always" or "occasionally") use of two, or occasionally three, acoustic properties (most frequently, amplitude and pace) that differed across listeners. This is logical since, when a motivated listener initially achieves good accuracy by using particular acoustic properties, that listener is likely to stay with his or her listening strategy; accuracy should then be high for the walkers for whom the attended properties are posture diagnostic. The least accurate listeners tended to individually report a broader range of acoustic property use, along with a lower frequency of use ("occasionally" or "usually") of any acoustic property. This pattern of least accurate listener reports most likely reflects either low motivation for the task or high motivation coupled with largely unsuccessful sampling of alternative decision strategies.

Although introspective self-reports need to be interpreted with caution, the results from the behavioral aspect of the present study are all consistent with the earlier analyses that indicated that posture-diagnostic information differed across walkers, as well as with the expectation that listeners, when faced with complex decision tasks with highly variable information content, will differ in decision strategies. In a sense, we have demonstrated the problems that we had anticipated in our initial contrast of challenges faced by investigations of natural acoustic events versus highly controlled auditory decision stimuli. However, the careful analyses of the source-event and sound information have provided a level of understanding of important source-sound relationships, as well as research tools that can begin to address listener strategies more directly, and

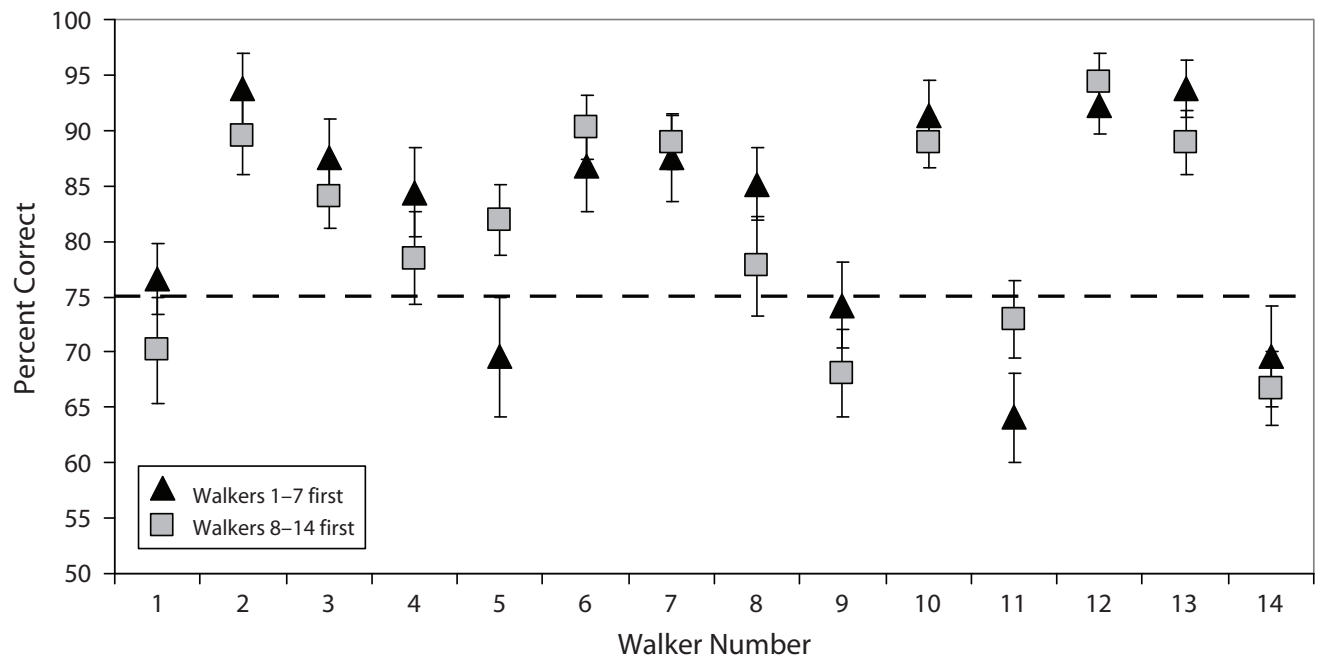

Figure 1. Average by-walker listener performance (experienced performance and generalization blocks) by listener group (Walkers 1-7 vs. Walkers 8-14 first). 
do so in the absence of the contrived and limited conditions typical of many laboratory studies.

Modeling listener use of information. Our previous modeling identified the predictive quality of the information available in the acoustic stimuli. Similar modeling can be used to evaluate listener use of acoustic information in performing behavioral tasks (Tanner, 1960). The strategy is to compare the pattern of accuracy across walkers predicted by the specific property models with the pattern exhibited by human listeners.

The performance of the hierarchical models depended on the criteria for each parameter, and optimum performance using the more predictive acoustic properties exceeded the accuracy of most listeners. With less-thanoptimum criteria, not only was model accuracy reduced, but so was the pattern of accuracy across walkers. Since the minimum level of accuracy for the better listeners was .75 , we began our listener evaluations by analyzing the matrix of total possible accuracy calculations from the two-property hierarchical models in order to determine sets of criteria that would produce .75 accuracy. The resulting patterns of across-walker accuracy were then correlated with the behavioral performance of individual listeners. Some of the two-property models produced strong correlations with a few individual listeners. For example, the data from Listener 53 were highly correlated $[r(55)=$ $.82, p<.05]$ with results of a hierarchical model that used amplitude followed by spectral slope. We next modified the criteria in the given models to match the overall accuracy for some of the individual listeners, and several of the comparisons produced high correlations [e.g., $r(55)=$ $.90, p<.05$, for Listener 25]. The best pattern matches were often observed for a different set of acoustic properties than were reported by the given listener. Thus, in addition to using a limited subset of information, it appears that listeners may have difficulty in accurately identifying their decision strategies.

\section{CONCLUDING COMMENTS}

The impact sounds from human gait reflect the properties of the shoe, the walking surface, and the force vectors at impact. A theoretical examination of the biomechanics of gait in the upright posture and measurements of source attributes indicate a complex coordinated motion system, with biomechanical couplings among the many body components and many differences among normal walkers, some relating to anthropometrical attributes and some simply reflecting learned mechanics. It is the combination of these factors that defines the impact forces. Because of this complexity, identical force vectors could be produced by different combinations of source properties, with the analogy being color metamers. In addition, because walking requires coordination of many widely distributed parts of the body, the impact forces exhibited by an individual walker will vary somewhat across individual steps. As a result, there is wide variability in the distribution of attributes of the sounds produced when the shoe impacts the walking surface. Therefore, properties of sounds produced during normal upright gait can be ex- pected to be complex and variable. Thus, although one can summarize the modal value of each sound attribute, and thus create a specific pattern of values across a subset of attributes, this information does not define a crisp, meaningful acoustic prototype for normal gait by healthy walkers in the upright posture. Instead, the complexity of the source event requires that something approximating an acoustic prototype be defined as probabilistic (or "fuzzy") weighted subsets of acoustic attributes.

Adjustment of gait to a stooped posture also is complex, differs across walkers, and is probably somewhat inconsistent for an individual walker. Hence, the changes in impact forces and the resulting acoustic properties are complex and variable rather than simple and invariant. It thus is unreasonable to expect there to be invariant acoustic cues that characterize gait in either posture, or that differentiate postures. At the same time, there is statistical evidence in the pattern of sounds that does differentiate walker posture, and humans, as complex decision makers, can access that information to make reasonable, and sometimes highly accurate, judgments of posture.

The human listener is the third corner of the source information triangle. Had there been one invariant cue that differentiated the posture contrast, the use of that cue should have been evident, both as a dichotomy in accuracy between listeners who used and did not use that cue and in the pattern of accuracy across the better-performing listeners. Instead, the present listeners differed widely in their overall ability to differentiate source postures, in patterns of accuracy across walkers, and in probable use of the available acoustic information; these findings are consistent with the reports summarized in the introduction.

Any investigation of the information triangle will operate with an implicit, if not an explicit, model of the source-sound-perception relationships, and the questions asked and the analyses employed will be guided, if not determined, by that model. A model that assumes invariant properties would operate in a manner very different from the complex one we have just described. Had the present study been based on an invariant-cue model, the outcome would have been negative; if gait were studied extensively with similar outcomes, one might even consider arguing that human gait perception involves special types of processing. Having begun with a very different model of the source-perception loop, the study has started to develop both a solid, empirically based picture of human auditory gait perception and a set of analysis tools to use with this type of loop model.

The source-perception variability model in the present study was motivated by consideration of the speech literature, but it considered how one should investigate other classes of complex natural events, those not assumed to be mediated by special processes in which invariant cues might or might not exist (CHABA, 1989). With the benefit of having the results from this nonspeech study, we now again consider the study of speech. There are similarities between speech and human gait as source events, since they are dynamic, complex, and coordinated; differ in many ways across the individuals producing the sounds; and differ even within individuals across events. 
This broad depiction applies to specific event classes (e.g., to gait in an upright posture or to production of the voiced labial stop consonant), as does the individual variability across specific contrasts (e.g., a posture contrast or a labial vs. velar stop). Speech differs from human gait in the nature of the complex sounds produced (e.g., speech is typically more continuous and has more different types of sounds). The mapping from source to sound across these events may differ somewhat in complexity, but both mappings are complex and variable. For speech, there is an extensive literature on the source event and the sound produced, and thus a far better understanding of these components of the source loop.

The last corner of the information triangle is the listener. Even if the human listener is wired for speech, is the wiring identical across speakers with the same native language? If our conceptualization of the source loop for natural sounds is applicable to speech, then research needs to be driven by a different conceptual model that can build on the excellent mapping of the source properties.

The broad conceptual model we have described and the analysis tools developed within the framework of this model are compatible with different theoretical perspectives. If, as posited by the Gibsonian approach, the dynamic structure in a sound reflects the dynamic structure in its source, this conceptual framework should identify both types of structure and the relationship between them. Furthermore, the analyses should indicate whether the sound structures that support source perception are similar or vary considerably across human listeners. Because the conceptual model for source perception seeks to identify the important acoustic information that listeners can use to make judgments about the source, as well as the tendency of listeners to use different pieces of information, it provides the framework needed for meaningful psychophysical investigation. More focused psychophysical investigations then can address meaningful questions about the importance of specific variables. The ultimate goal of this broad perspective on source perception should be to develop a thorough understanding of the nature and relationships among source, sound, and perception, with theoretical beliefs developing from that understanding rather than driving the research.

\section{AUTHOR NOTE}

The authors thank Robert Downes for his comments and suggestions concerning the biomechanics of human gait, which were extremely helpful in making sense of the source event and its implications for sound and perception. They also thank Massimo Grassi and Steven Handel for their extremely useful comments and suggestions on earlier drafts of the manuscript. This material is based on work supported by the National Science Foundation under Grant 0213666. Any opinions, findings, and conclusions or recommendations expressed in this material are those of the authors and do not necessarily reflect the views of the National Science Foundation. Correspondence related to this article should be addressed to R. E. Pastore, Department of Psychology, Binghamton University, Binghamton, NY 13902-6000 (e-mail: pastore@binghamton.edu).

\section{REFERENCES}

Ballas, J. A. (1993). Common factors in the identification of an assortment of brief everyday sounds. Journal of Experimental Psychology: Human Perception \& Performance, 19, 250-267.
Ballas, J. A., \& Mullins, T. (1991). Effects of context on the identification of everyday sounds. Human Performance, 4, 199-219.

Braune, W., \& Fischer, O. (1987). The human gait (P. Maquet \& R. Furlong, Trans.). Berlin: Springer. (Original work published 1899)

Broer, M. B. (1973). Efficiency of human movement. Philadelphia: W. B. Saunders.

CAPPOZZO, A. (1991). The mechanics of human walking. In A. E. Patla (Ed.), Adaptability of human gait: Implications for the control of locomotion (pp. 167-186). Amsterdam: North-Holland.

CHABA (Committee on Hearing, Bioacoustics, \& Biomechanics, National Research Council) (1989). Classification of complex nonspeech sounds. Washington, DC: National Academy Press.

CutTing, J. E. (1978). Generation of synthetic male and female walkers through manipulation of a biomechanical invariant. Perception, 7, 393-405.

Cutting, J. E., \& Kozlowski, L. T. (1977). Recognizing friends by their walk: Gait perception without familiarity cues. Bulletin of the Psychonomic Society, 9, 353-356.

Cutting, J. E., Proffitt, D. R., \& Kozlowski, L. T. (1978). A biomechanical invariant for gait perception. Journal of Experimental Psychology: Human Perception \& Performance, 4, 357-372.

FARRINGTON, S. M. (1998). Invariant acoustic cues for determining source characteristics of footstep sounds. Unpublished master's thesis, State University of New York, Binghamton.

Fowler, C. A. (1991). Auditory perception is not special: We see the world, we feel the world, we hear the world. Journal of the Acoustical Society of America, 89, 2910-2915.

Fowler, C. A., \& Rosenblum, L. D. (1991a). Duplex perception: A comparison of monosyllables and slamming doors. Journal of Experimental Psychology: Human Perception \& Performance, 16, 742-754.

Fowler, C. A., \& Rosenblum, L. D. (1991b). Perception of the phonetic gesture. In I. G. Mattingly \& M. Studdert-Kennedy (Eds.), Modularity and the motor theory of speech perception (pp. 33-59). Hillsdale, NJ: Erlbaum.

FreED, D. J. (1990). Auditory correlates of perceived mallet hardness for a set of recorded percussive sound events. Journal of the Acoustical Society of America, 87, 311-322.

Galbraith, F. W., \& Barton, M. V. (1970). Ground loading from footsteps. Journal of the Acoustical Society of America, 48, 1288-1292.

GAVER, W. W. (1993). What in the world do we hear? An ecological approach to auditory event perception. Ecological Psychology, 5, 1-29.

GiBson, J. J. (1966). The senses considered as perceptual systems. Boston: Houghton Mifflin.

GiBson, J. J. (1979). The ecological approach to perception. Boston: Houghton Mifflin.

Giordano, B. L., \& McAdams, S. (2006). Material identification of real impact sounds: Effects of size variation in steel, glass, wood, and plexiglass plates. Journal of the Acoustical Society of America, 119, 1171-1181.

Grassi, M. (2005). Do we hear size or sound? Balls dropped on plates. Perception \& Psychophysics, 67, 274-284.

Green, D. M., \& Swets, J. A. (1966). Signal detection theory and psychophysics. New York: Wiley.

GyGI, B. (2001). Factors in the identification of environmental sounds. Unpublished doctoral dissertation, Indiana University.

HANDEL, S. (1989). Listening: An introduction to the perception of auditory events. Cambridge, MA: MIT Press.

HANDEL, S. (1995). Timbre perception and auditory object recognition. In B. C. J. Moore (Ed.), Hearing (pp. 425-461). New York: Academic Press.

Hartmann, W. M. (1998). Signals, sound, and sensation. Berlin: Springer.

Howard, J. H., \& Ballas, J. A. (1980a). Perception of simulated propeller cavitation. Human Factors, 25, 643-656.

HowARD, J. H., \& BaLlas, J. A. (1980b). Syntactic and semantic factors in the classification of nonspeech transient patterns. Perception \& Psychophysics, 28, 431-439.

Howard, J. H., \& Ballas, J. A. (1981). Feature selection in auditory perception. In D. J. Getty \& J. H. Howard (Eds.), Auditory and visual pattern recognition (pp. 181-196). Hillsdale, NJ: Erlbaum.

JACOBS, A., \& Shiffrar, M. (2005). Walking perception by walking observers. Journal of Experimental Psychology: Human Perception \& Performance, 31, 157-169. 
Jenkins, J. J. (1985). Acoustic information for objects, places, and events. In W. H. Warren \& R. E. Shaw (Eds.), Persistence and change: Proceedings of the First International Conference on Event Perception (pp. 115-138). Hillsdale, NJ: Erlbaum.

Johansson, G. (1973). Visual perception of biological motion and a model for its analysis. Perception \& Psychophysics, 14, 201-211.

Johansson, G. (1986). Relational invariance and visual space perception: On perceptual vector analysis of the optic flow. Acta Psychologica (Special issue: Sensorimotor interactions in space perception and action), 63, 89-101.

Kunkler-Peck, A. J., \& Turvey, M. T. (2000). Hearing shapes. Journal of Experimental Psychology: Human Perception \& Performance, 26, 279-294.

Lakatos, S., Gibson, G., \& Cannon, R. (1997, November). Mental representation of sound sources: Interaction between source and exciter. Paper presented at the 38th Annual Meeting of the Psychonomic Society, Philadelphia.

Lakatos, S., McAdams, S., \& CAussé, R. (1997). The representation of auditory source characteristics: Simple geometric form. Perception \& Psychophysics, 59, 1180-1190.

Li, X.-F., Logan, R. L., \& Pastore, R. E. (1991). Perception of acoustic source characteristics: Walking sounds. Journal of the Acoustical Society of America, 90, 3036-3049.

Liberman, A. M., \& Mattingly, I. G. (1985). The motor theory of speech perception revisited. Cognition, 21, 1-36.

LuCE, R. D. (1963a). A threshold theory for simple detection experiments. Psychological Review, 70, 61-79.

LutFI, R. A., \& OH, E. L. (1997). Auditory discrimination of material changes in a struck-clamped bar. Journal of the Acoustical Society of America, 102, 3647-3656.

Macmillan, N. A., \& Creelman, C. D. (1991). Detection theory: A user's guide. Cambridge: Cambridge University Press.

Macmillan, N. A., \& Creelman, C. D. (2005). Detection theory: A user's guide (2nd ed.). Mahwah, NJ: Erlbaum.

Macmillan, N. A., \& Kingston, J. (1995). Integrality of nasalization and F-sub-1 in vowels in isolation and before oral and nasal consonants: A detection-theoretic application of the Garner paradigm. Journal of the Acoustical Society of America, 97, 1261-1285.

McADAMs, S. (1984). The auditory image: A metaphor for musical and psychological research on auditory organization. In W. R. Crozier \& A. J. Chapman (Eds.), Cognitive processes in the perception of art (pp. 289-323). Amsterdam: North-Holland.

McAdams, S. (1993). Recognition of sound sources and events. In S. McAdams \& E. Bigand (Eds.), Thinking in sound: The cognitive psychology of human audition (pp. 146-198). Oxford: Oxford University Press, Clarendon Press.

McAdams, S., Chaigne, A., \& Roussarie, V. (2004). The psychomechanics of simulated sound sources: Material properties of impacted bars. Journal of the Acoustical Society of America, 115, 1306-1320.

Mirman, D., Holt, L. L., \& McClelland, J. L. (2004). Categorization and discrimination of nonspeech sounds: Differences between steadystate and rapidly-changing acoustic cues. Journal of the Acoustical Society of America, 116, 1198-1207.

Moore, B. C. J. (2003). Introduction to the psychology of hearing. New York: Academic Press.

National Center for Health Statistics (updated 2003). [Anthropometric reference data, United States, 1988-1994]. Retrieved March 16, 2005, from www.cdc.gov/nchs/fastats/bodymeas.html.

Pastore, R. E., Crawley, E. J., Berens, M. S., \& Skelly, M. A. (2003). "Nonparametric" $A$ ' and other modern misconceptions about signal detection theory. Psychonomic Bulletin \& Review, 10, 556-569.

PitT, M. A. (1994). Perception of pitch by musically trained and untrained listeners. Journal of Experimental Psychology: Human Perception \& Performance, 20, 976-986

Proffitt, D. R., \& KaIser, M. K. (1995). Perceiving events. In W. Epstein \& S. Rogers (Eds.), Perception of space and motion (pp. 228262). New York: Academic Press.

Raphael, L. J. (2005). Acoustic cues to the perception of segmental phonemes. In D. B. Pisoni \& R. E. Remez (Eds.), The handbook of speech perception (pp. 182-206). Malden, MA: Blackwell.

REPP, B. H. (1987). The sound of two hands clapping: An exploratory study. Journal of the Acoustical Society of America, 81, 1100-1110.

Runeson, S., \& FryKholm, G. (1983). Kinematic specification of dy- namics as an informational basis for person-and-action perception: Expectation, gender recognition, and deceptive intention. Journal of Experimental Psychology: General, 112, 585-615.

Samoylenko, E., McAdams, S., \& Nosulenko, V. (1996). Systemic analysis of verbalizations produced in comparing musical timbres. International Journal of Psychology, 31, 255-278.

TANNER, W. P., JR. (1960). Theory of signal detectability as an interpretive tool for psychological data. Journal of the Acoustic Society of America, 32, 1140-1147.

TANNER, W. P., JR. (1961). Physiological implications of psychophysical data. Annals of the New York Academy of Sciences, 89, 752-765.

VANDERVEER, N. J. (1980). Ecological acoustics: Human perception of environmental sounds. Dissertation Abstracts International, 40(9-B), 4543.

WALD, A. (1955). Basic ideas of a general theory of statistical decision rules. In T. W. Anderson \& the Institute of Mathematical Statistics (Eds.), Selected papers in statistics and probability, by Abraham Wald (pp. 656-668). New York: McGraw-Hill.

Warren, W. H., \& VerbrugGe, R. R. (1984). Auditory perception of breaking and bouncing events: A case study in ecological acoustics. Journal of Experimental Psychology: Human Perception \& Performance, 10, 704-712.

Weber, W., \& WeBer, E. (1992). Mechanics of the human walking apparatus (P. Maquet \& R. Furlong, Trans.). Berlin: Springer. (Original work published in 1836)

Werker, J. F., \& TeEs, R. C. (1999). Influences on infant speech processing: Toward a new synthesis. Annual Review of Psychology, 50, 509-535.

WERnICK, J. (1993). The E.D.G. system for dynamic analysis of gait. Clinics in Podiatric Medicine \& Surgery, 10, 431-443.

WiLDES, R., \& RichARDS, W. (1988). Recovering material properties from sound. In W. Richards (Ed.), Natural computation (pp. 256-363). Cambridge, MA: MIT Press.

WinTER, D. A. (1987). The biomechanics and motor control of human gait. Waterloo, ON: University of Waterloo Press.

Winter, D. A. (1989). Coordination of motor tasks in human gait. In S. A. Wallace (Ed.), Perspectives on the coordination of movement (pp. 329-376). Amsterdam: North-Holland.

Yamaguchi, G. T., Pandy, M. G., \& Zajac, F. E. (1991). Dynamic musculoskeletal modeling of human locomotion: Perspectives on model formulation and control. In A. E. Patla (Ed.), Adaptability of human gait: Implications for the control of locomotion (pp. 205-240). Amsterdam: North-Holland.

Yost, W. A. (1994). Fundamentals of hearing. San Diego: Academic Press.

ZATSIORSKy, V. M. (1998). Kinematics of human motion. Champaign, IL: Human Kinetics.

\section{NOTES}

1. Several additional walkers were also recorded, but because of abnormal gait (e.g., toe strike before heel strike), the stimuli from these walkers were not processed.

2. A second set of simultaneous DAT recordings were made with an identical microphone on a 5-foot floor stand located 3 feet from the center of the floor. Because of greater variation in amplitude and lower signal-to-noise ratio, these recordings were not used in this study. Neither of the Sony DAT units was capable of direct digital input to a computer.

3. A few walkers wore longer pants of denim material that tended to add friction-produced sounds that had the potential to differ across postures. To ensure that listeners in the later behavioral analysis would use only the impact sounds, and not these extraneous noises, we decided not to use the stimuli from these walkers in the present study.

4 . Because the independent-measures $t$ tests for the other biomechanical measures were not significant, we do not report them here. They are available on our Web site, www.bingweb.binghamton.edu/ pastore.

5. Goodness of fit was not calculated for spectral slope (linear).

6. Spectral centroid for heel impact sounds, $t(26)=1.7, p=.10$; spectral centroid for sole impact sounds, $t(26)=0.82, p=.42$; spectral slope for heel impact sounds, $t(26)=-2.1, p=.04$.

7 . In addition to computing $d^{\prime}$ from standard deviations averaged across postures, we also computed $d_{\mathrm{s}}^{\prime}$, which does not assume equal 
variance. The values of $d^{\prime}$ and $d_{\mathrm{s}}^{\prime}$ for each acoustic property, as well as the trends in values across walkers, were similar.

8. This analysis assumed for each walker the same decision mapping as the across-walker analysis for the given property. Specifically, if the across-walker mean for the property was larger for upright posture, $d^{\prime}$ was positive for a given walker when the walker's upright mean was larger than the stooped mean, and $d^{\prime}$ was negative when the walker's stooped mean was greater. Thus, for some walkers $p(\mathrm{c})_{\max }$ was less than chance.

9. Pitt (1994) suggested that nonmusicians may easily confuse the dimensions of pitch and timbre. We thus asked participants to rate their use of "pitch" rather than timbre, but further described pitch as a rating of the quality of the sound (low, high, full, etc.).
10. If we assume a high-threshold model (see, e.g., Macmillan \& Creelman, 1991, 2005) and that incorrect responses are due to guessing, an average listener accuracy of .69 would mean that only $38 \%$ of the answers were based on relevant information that led to a correct answer, so that the average listener guessed for the other $62 \%$ of trials. The minimum accuracy criterion we adopted, .75 , represents half of the trials being based on stimulus information, coupled with correct guessing on half of the remaining trials.

(Manuscript received June 2, 2005;

revision accepted for publication June 13, 2007.) 